\title{
RADIO EMISSION IN PECULIAR GALAXIES
}

\author{
Dulia F. de Mello Rabaça and Zulema Abraham \\ Instituto Astronomico e Geofisico - USP \\ C.P. 30627, 01051, Sao Paulo, Brazil
}

During the last decades a number of surveys of peculiar galaxies have been carried out and accurate positions become available. Since peculiarities are a possible evidence of radio emission (Wright, 1974; Sulentic, 1976; Stocke et al.,1978), we have selected a sample of 24 peculiar galaxies with optical jet-like features or extensions in different optical catalogues, mainly the Catalogue of Southern Peculiar Galaxies and Associations (Arp and Madore, 1987) and the ESO/Uppsala Survey of the ESO(B) Atlas (Lauberts, 1982) for observation at the radiocontinuum frequency of $22 \mathrm{GHz}$. The sample is listed in the Table.

Sol (1987) studied this sample and concluded that the majority of the jet-like features seem to admit an explanation in terms of interactive galaxies with bridges and/or tails due to tidal effects. Only in few cases the "jets" seem to be possibly linked to some nuclear activity of the host galaxy.

The observations were made with the $13.7 \mathrm{~m}$-radome enclosed Itapetinga Radiotelescope (HPBW of 4.3 arcmin), in Brazil. The receiver was a $1 \mathrm{GHz}$ d.s.b. super-heterodine mixer operated in total-power mode, with a system temperature of aproximately $800 \mathrm{~K}$. The observational technique consisted in scans in right ascention, centralized in the optical position of the galaxy. The amplitude of one scan was 43 arcmin, and its duration time was 20 seconds. The integration time was at least 2 hours (12 ten-minute observations) and the sensibility limit adopted was an antenna temperature greater than 3 times the r.m.s. error of the baseline determination. Virgo A was used as the calibrator source.

Three galaxies were detected for the first time as radio sources and four other known galaxies at low frequencies had their flux densities measured at $22 \mathrm{GHz}$. The results for these sources are presented in the figures (a) to (f). 
Sample of Peculiar Galaxies

\begin{tabular}{|c|c|c|c|c|c|}
\hline SOURCE & DESIG & $\begin{array}{l}\text { R.A. (1950) } \\
\text { h m s }\end{array}$ & $\begin{array}{c}\text { DEC. }(1950) \\
\text { o }, "\end{array}$ & PECULIARIT & \\
\hline 0113-52 & & 011306 & -525500 & jet & (S) \\
\hline $0145-48$ & & 014506 & -483900 & tail & (S) \\
\hline $0147-27$ & 414IG4 & 014713 & -275654 & tail & (L) \\
\hline $0149-48$ & & 014930 & -485100 & jet & $(\mathrm{AM})$ \\
\hline $0207-49$ & 197IG25 & 020659 & $-4931 \quad 18$ & jet & $(\mathrm{AM})$ \\
\hline 0214-26 & $478 \mathrm{G} 19$ & 021403 & $\begin{array}{llll}-26 & 10 & 54\end{array}$ & streamer & $(\mathrm{L})$ \\
\hline $0229-58$ & $115 \mathrm{G} 15\left(^{*}\right)$ & 022941 & $\begin{array}{llll}-58 & 08 & 18\end{array}$ & $\mathrm{~S} 0$ in $\mathrm{cl}$ & (L) \\
\hline 0256-36 & $356 \mathrm{IG} 24$ & 025656 & -364848 & jet & (L) \\
\hline 0339-54 & $156 \mathrm{IG} 7$ & 033956 & -540957 & streamer & (L) \\
\hline $0349-27$ & $(*)$ & 034932 & -275332 & jet & (C) \\
\hline 0404-52 & 156G38 & 040452 & -524748 & b dwarf & (S) \\
\hline $0422-51$ & $202 \mathrm{G} 14$ & 042230 & -514248 & jet & $(\mathrm{AM})$ \\
\hline $0426-55$ & 157G32 & 042644 & -550940 & interact & (S) \\
\hline $0430+05$ & $3 \mathrm{C} 120\left(^{*}\right)$ & 043030 & +051500 & jet & $(\mathrm{AM})$ \\
\hline 0521-36 & $\left({ }^{*}\right)$ & 052113 & $\begin{array}{lll}-36 & 30 & 16\end{array}$ & jet & (S) \\
\hline 0606-29 & $425 \mathrm{G} 9$ & 060637 & -293200 & jet & (L) \\
\hline 0646-64 & 87IG41 & 064637 & -645412 & pair & (L) \\
\hline $2030-66$ & & 203018 & -662600 & jet & $(\mathrm{AM})$ \\
\hline $2110-61$ & $144 \mathrm{G} 20$ & 210955 & -612948 & jet & $(\mathrm{AM})$ \\
\hline $2155-69$ & $75 \mathrm{G} 45$ & 215506 & -694330 & jet & $(\mathrm{AM})$ \\
\hline $2207-67$ & 108IG17 & 220700 & -670654 & jet & $(\mathrm{AM})$ \\
\hline $2300+16$ & & 230030 & +162000 & $\mathrm{Mkr}$ & $(\mathrm{S})$ \\
\hline $2329-41$ & & 232924 & -410000 & jet & $(\mathrm{AM})$ \\
\hline 2331-38 & $347 \mathrm{G} 22$ & 233049 & -385254 & jet & $(\mathrm{AM})$ \\
\hline
\end{tabular}

Columns are: (1) galaxy name; (2) ESO/Uppsala designation, an asterisk indicates radio emission in low frequencies; (3)-(4) equatorial coordinate s; (5) peculiarities; and (6) references: (S) Sol (1987), (L) Lauberts (1982), (AM) Arp and Madore (1987), and (C) Christiansen (1977) 

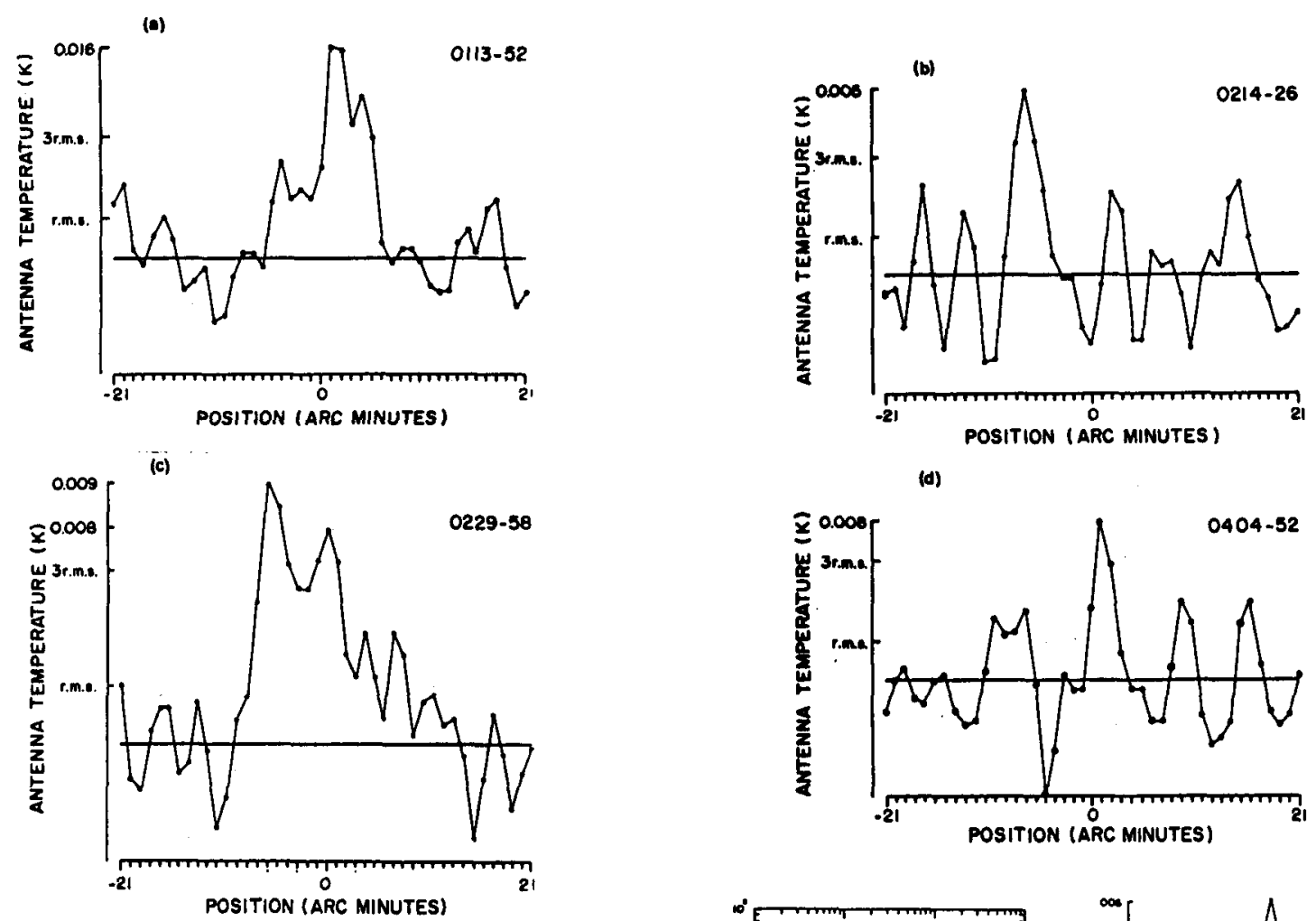

(d)
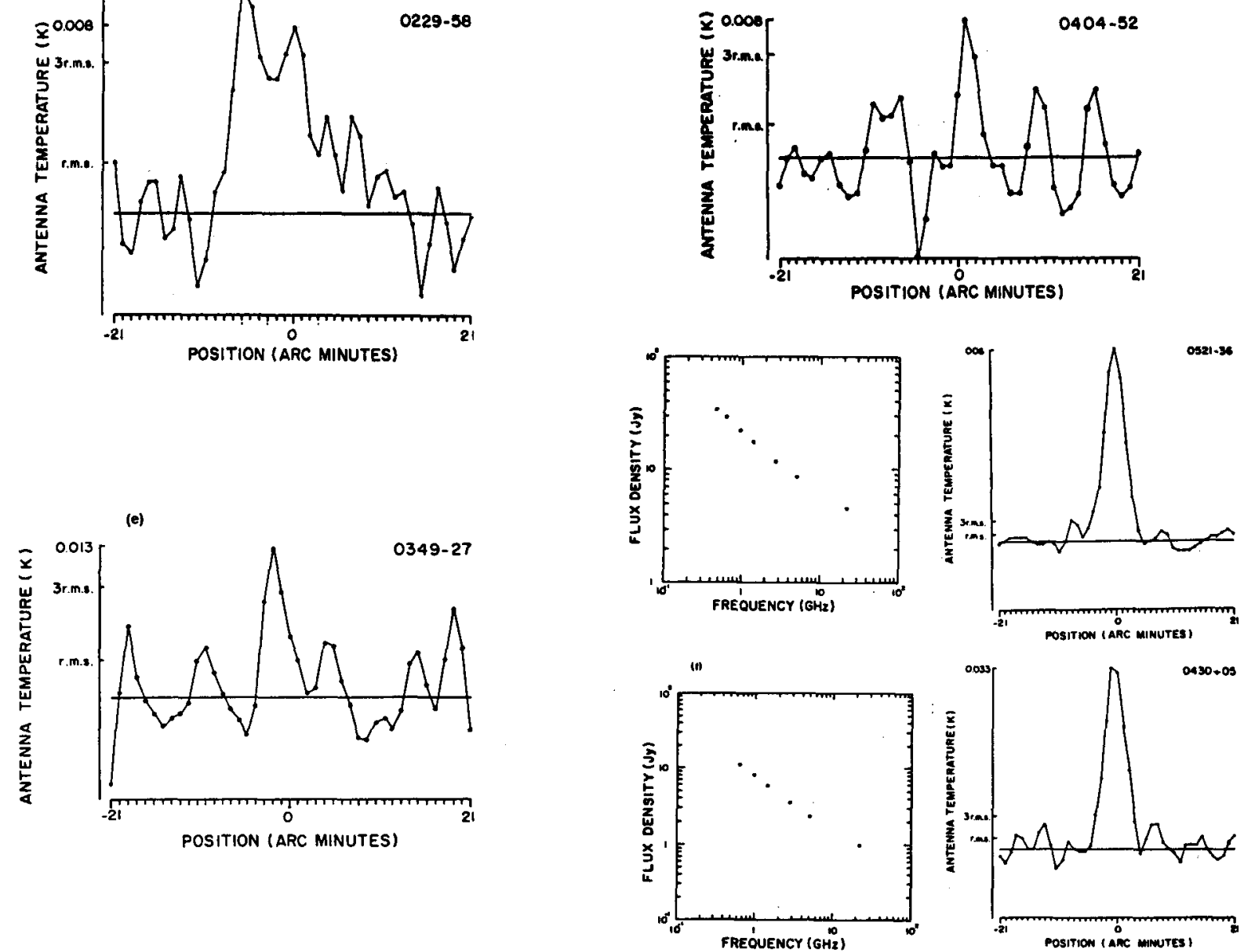

Figures are: (a) 0113-52 - The radio source position coincides with the optical galaxy. The flux density is $(1.2 \pm 0.2) \mathrm{Jy}$. (b) 0214-26 - The radio source is shifted 7 arcmin from the optical galaxy. Since the redshift of this object is not known, it is not possible to calculate the actual distance between the radio and optical sources. If we suppose a common redshift for galaxies like 0.02 , the distance will be $200 \mathrm{Kpc}$, a typical value between the radio source and the optical counterpart. The flux density is $(0.4 \pm 0.1) \mathrm{Jy}$. 
(c) 0229-58 - The radio source is about 6 arcmin shifted from the galaxy. Using a radial velocity of $9590 \mathrm{Km} / \mathrm{s}$ (Lauberts, 1982) for calculation, the distance between the optical position and the maximum of the radio emission is $335 \mathrm{Kpc}$. The maximum flux density is $(0.7 \pm 0.2) \mathrm{Jy}$. (d) 0349-27 - The radio source position coincides with the optical galaxy. The flux density is $(1.0 \pm 0.2) \mathrm{Jy}$. This source has fluxes measured at low radio frequencies (Wills, 1975) and a typical non-thermal steep-spectrum. (e) 0404-52 - The radio source coincides with the optical galaxy. The flux density is $(0.6 \pm 0.2) \mathrm{Jy}$. (f) $0430+05$ and $0521-36$ - Both galaxies are well known radio sources at low radio frequencies. The flux densities at $22 \mathrm{GHz}$ are $(2.5 \pm 0.2) \mathrm{Jy}$ and $(4.5 \pm 0.2) \mathrm{Jy}$, respectively. The spectrum is non-thermal in both cases, and their radio emission coincides with the optical position.

\section{ACKNOWLEDGEMENTS}

We would like to thank Helene Sol for the sample and suggestions and Carlos R. Rabaca for many comments. This work was partially supported by the Brazilian agencies $\mathrm{CNPq}$ and FAPESP.

\section{REFERENCES}

Arp, H.; Madore, B.F. (1987) A Catalogue of southern peculiar galaxies and associations. University of Toronto.

Christiansen, W.N.; Frater, R.H.; Watkinson, A.; O'Sullivan, J.D.; Lockhart, I.A.; Goss, W.M. (1977) M.N.R.A.S., 181, 183.

Lauberts, A. (1982) The ESO/Uppsala Survey of the ESO(B) Atlas.

Sol, H. (1987) Jets et sources radio extragalactiques. These de Doctorat D'Etat, Universite de Paris VII.

Stocke, J.T.; Tiftt, W.G.; Kaftan-Kassim, M.A. (1978) Astron. J., 83, 322.

Sulentic, J.W. (1976) Astrophys. J. Suppl. Series, 32, 171.

Wright, A.E. (1972) M.N.R.A.S., 167, 251. 